\title{
Video Article \\ Remote Sensing Evaluation of Two-spotted Spider Mite Damage on Greenhouse Cotton
}

\author{
Daniel E. Martin ${ }^{1}$, Mohamed A. Latheef ${ }^{1}$ \\ ${ }^{1}$ USDA-ARS, SPARC-AATRU \\ Correspondence to: Daniel E. Martin at daniel.martin@ars.usda.gov \\ URL: https://www.jove.com/video/54314 \\ DOI: doi:10.3791/54314
}

Keywords: Environmental Sciences, Issue 122, Spider mites, Tetranychus urticae, sampling spider mites, cotton, remote sensing, multispectral optical sensor, NDVI

Date Published: 4/28/2017

Citation: Martin, D.E., Latheef, M.A. Remote Sensing Evaluation of Two-spotted Spider Mite Damage on Greenhouse Cotton. J. Vis. Exp. (122), e54314, doi:10.3791/54314 (2017).

\section{Abstract}

The objective of this study was to evaluate a ground-based multispectral optical sensor as a remote sensing tool to assess foliar damage caused by the two-spotted spider mite (TSSM), Tetranychus urticae Koch, on greenhouse grown cotton. TSSM is a polyphagous pest which occurs on a variety of field and horticultural crops. It often becomes an early season pest of cotton in damaging proportions as opposed to being a late season innocuous pest in the mid-southern United States. Evaluation of acaricides is important for maintaining the efficacy of and preventing resistance to the currently available arsenal of chemicals and newly developed control agents. Enumeration of spider mites for efficacy evaluations is laborious and time consuming. Therefore, subjective visual damage rating is commonly used to assess density of spider mites. The NDVI (Normalized Difference Vegetation Index) is the most widely used statistic to describe the spectral reflectance characteristics of vegetation canopy to assess plant stress and health consequent to spider mite infestations. Results demonstrated that a multispectral optical sensor is an effective tool in distinguishing varying levels of infestation caused by $T$. urticae on early season cotton. This remote sensing technique may be used in lieu of a visual rating to evaluate insecticide treatments.

\section{Video Link}

The video component of this article can be found at https://www.jove.com/video/54314/

\section{Introduction}

Two-spotted spider mite, Tetranychus urticae (Koch) is a polyphagous and cosmopolitan pest of many field and horticultural plants ${ }^{1,2}$. It lives inside webbings in colonies on the bottom surface of the plant ${ }^{3,4}$. It has evolved from being a late season to an early season pest in the midsouthern United States over the last decade ${ }^{5}$. TSSM was the $5^{\text {th }}$ most damaging pest of cotton and caused an estimated loss of 57,441 bales of cotton and $0.167 \%$ reduction in yield in the United States in $2011^{6,7}$. Its short life cycle, high fecundity and haploid-diploid sex determination combined with the ability to digest and detoxify xenobiotics have exacerbated the development of resistance to pesticides ${ }^{8}$. Presently, acaricides remain as the only dependable control mechanism for the suppression of $T$. urticae. Therefore, field entomologists constantly evaluate currently available and newly developed acaricides for efficacy.

The estimation of damage by the spider mites is usually conducted by scoring the damage on a subjective scale because of the difficulty encountered in manually counting the mites. Some conducted binomial sampling, where only the proportion of infested leaves was scored rather than the number of spider mites per leaf ${ }^{9}$. A leaf reddening index scale, which varied from stippling and reddening to extensive reddening of the vegetation, was used as a criterion in estimating damage. The spatial distribution pattern of $T$. urticae on cotton conformed to a clumped distribution pattern ${ }^{9}$. Mites are distributed on cotton foliage from sparse to heavily clustered and remain so under field condition. Such distribution pattern coupled with its small size, mobility and prolific reproduction makes enumeration of TSSM difficult. Reliable alternative techniques are needed for the assessment of mite density in order to quantitatively evaluate efficacy of acaricides against TSSM.

The objective of this study was to separate cotton plants damaged by varying densities of TSSM by using an optical multispectral sensor. Our intent was to determine if the ground-based optical sensor could classify and separate healthy cotton plants from those damaged by the spider mites.

\section{Protocol}

\section{Establish TSSM Colonies on Pinto Beans}

1. Plant pinto beans, Phaseolus vulgaris L., in plastic trays $\left(56 \times 28 \times 5 \mathrm{~cm}^{3}\right)$ containing potting soil in the greenhouse as shown in Figure 1 .

2. Label the trays with marking sticks according to treatment and replication.

3. Set and maintain greenhouse temperature to $90{ }^{\circ} \mathrm{F}$ and $70 \%$ Relative Humidity. 
4. Grow beans to $1-2$ trifoliate leaf stage ${ }^{10}$ as shown in Figure 2.

5. Collect spider mites from cotton plants infested naturally with mites by removing infested leaves.

6. Place spider mite infested cotton leaves on pinto beans as often as necessary until all plants in the trays are infested with numerous TSSMs.

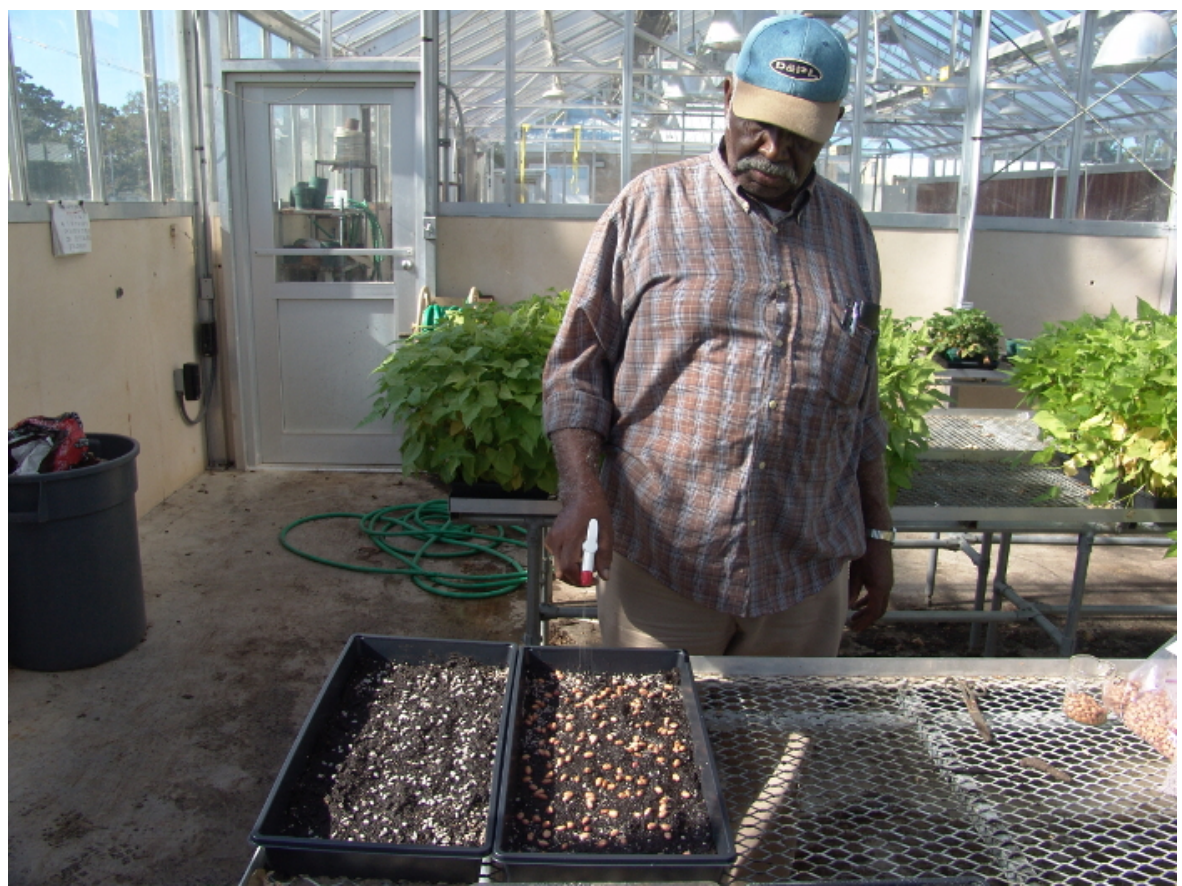

Figure 1: Planting pinto beans in plastic trays. Pinto beans seeds were planted in plastic trays $\left(56 \times 28 \times 5 \mathrm{~cm}^{3}\right)$ in the greenhouse and were watered daily. Please click here to view a larger version of this figure.

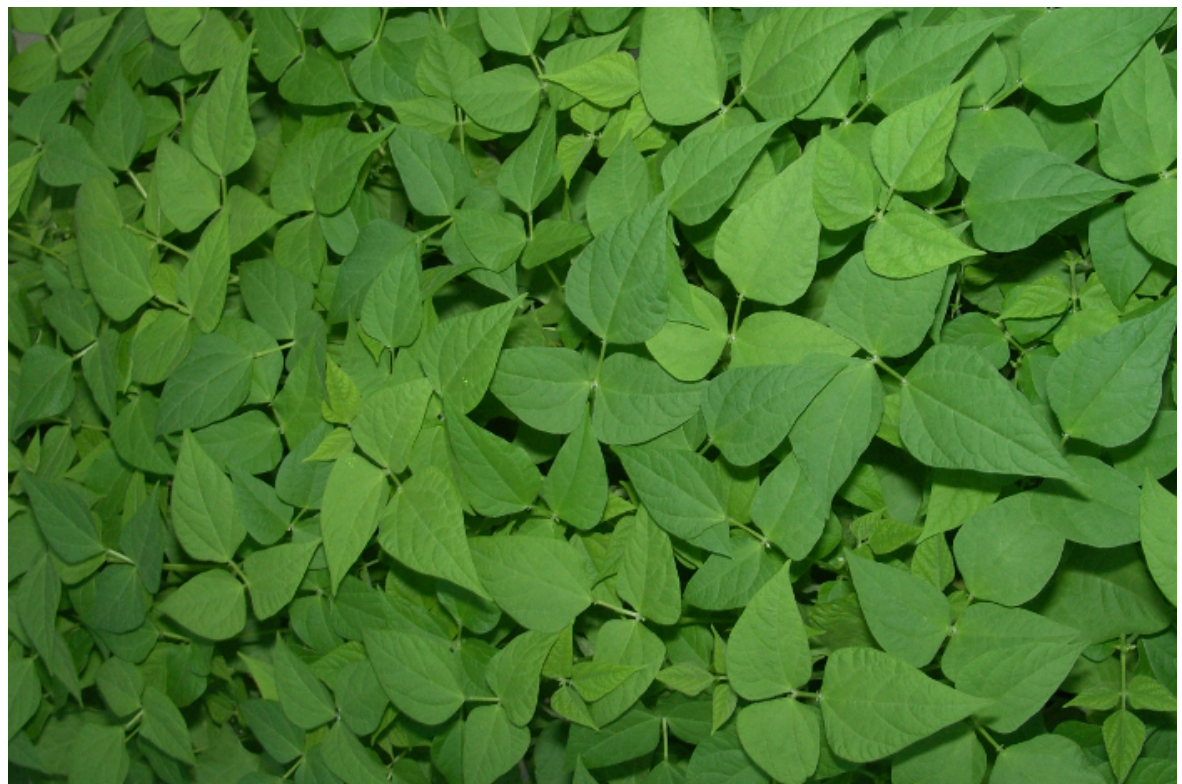

Figure 2: Pinto beans with trifoliate leaves. The first true leaf that is formed after the cotyledons emerge from the soil is the simple or unifoliate leaf. Subsequent leaves are the trifoliate leaves with denticulate tips. Please click here to view a larger version of this figure.

\section{Transfer TSSM to Cotton Plants}

1. Grow Non-Bt (not genetically modified) cotton plants to $4-5$ true leaf stage in plastic trays $(56 \times 28 \times 5 \mathrm{~cm})$ in the greenhouse as specified in step 1.3 and shown in Figure 3.

2. Transfer spider mite colonies from pinto beans onto young cotton plants at 4-5 true leaf stage.

1. Transfer 3 masses of spider mites for lightly infested plants. Note: At very high infestation levels, spider mites form masses or bolls ${ }^{11}$ and are found hanging on leaf tips as seen in Figure 4.

1. Place a pan under pinto bean leaf tip containing TSSM masses.

2. Cut pinto bean leaf tips with scissors, allowing TSSM masses to fall into the pan as shown in Figures $\mathbf{5}$ and $\mathbf{6}$. 
3. Turn pan upside down over cotton plants and tap TSSM masses onto cotton plants grown in plastic trays as shown in Figure 7 . Note: Each tray contained $\sim 100$ cotton plants.

4. Randomly spread 3 masses of TSSM onto cotton plants.

2. Transfer 20 masses for medially infested plants.

1. Place a pan under pinto bean leaf tip containing TSSM masses.

2. Cut pinto bean leaf tips with scissors, allowing them to fall into the pan.

3. Collect 20 masses in the pan.

4. Turn pan upside down over cotton plants and tap out TSSM masses onto $\sim 100$ cotton plants raised in the greenhouse.

3. Transfer 40 masses for heavily infested plants.

1. Place a pan under pinto bean leaf tip containing TSSM masses.

2. Cut pinto bean leaf tips with scissors, allowing them to fall into the pan.

3. Collect 40 masses in the pan.

4. Turn pan upside down over cotton plants, tap out TSSM masses onto $\sim 100$ cotton plants and spread them randomly.

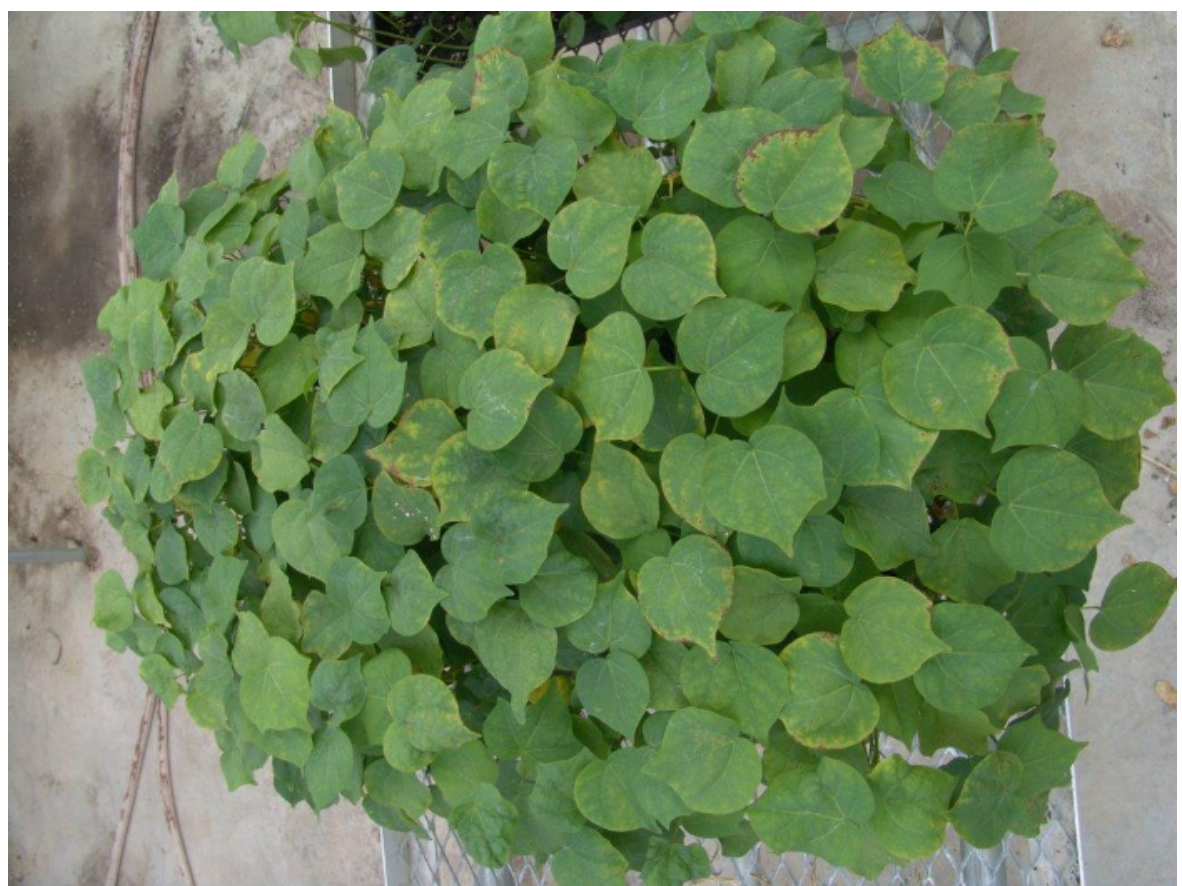

Figure 3. Cotton plants with 4-5 true leaf stage. The cotyledons emerge from the soil as leaf-like structures oriented opposite to each other on the seedling stem. The apical meristem emerges through the cotyledons and form the first true leaves. Please click here to view a larger version of this figure. 


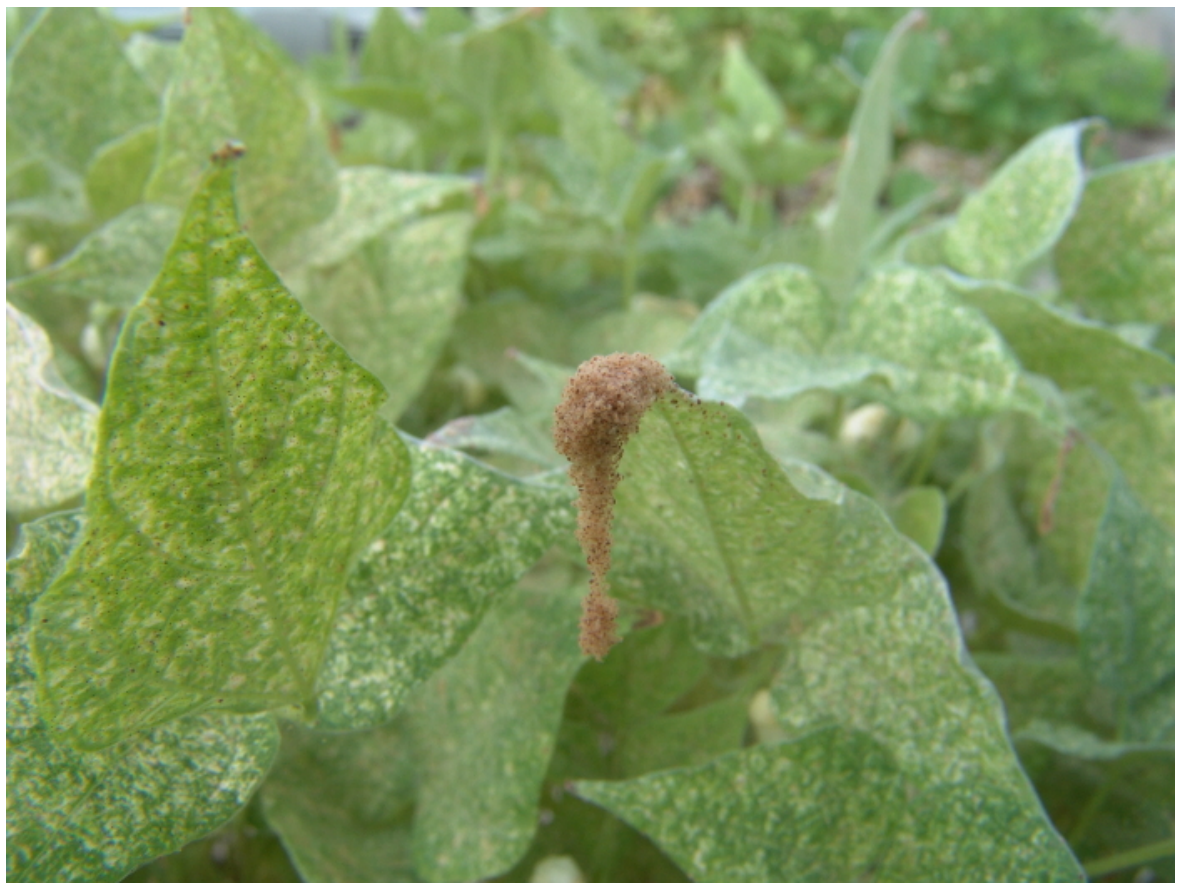

Figure 4. TSSM masses hanging on a trifoliate bean leaf. TSSM lives in colonies and when populations reach high density, they form masses or boll-like structures and congregate at the leaf tips for dispersal. Please click here to view a larger version of this figure.

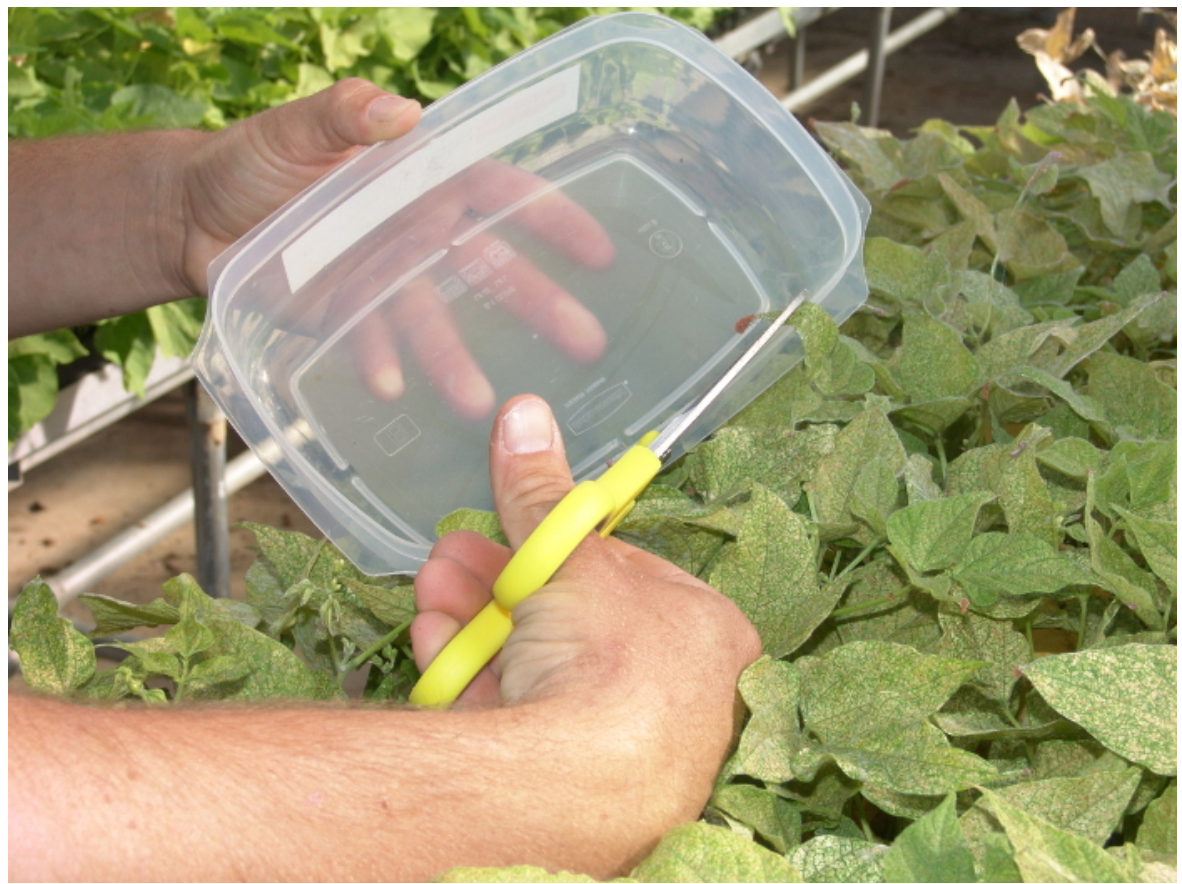

Figure 5. Cutting pinto bean leaf tips containing a TSSM mass with scissors. Trifoliate bean leaf tips containing TSSM masses were removed with scissors for infesting cotton plants. Please click here to view a larger version of this figure. 


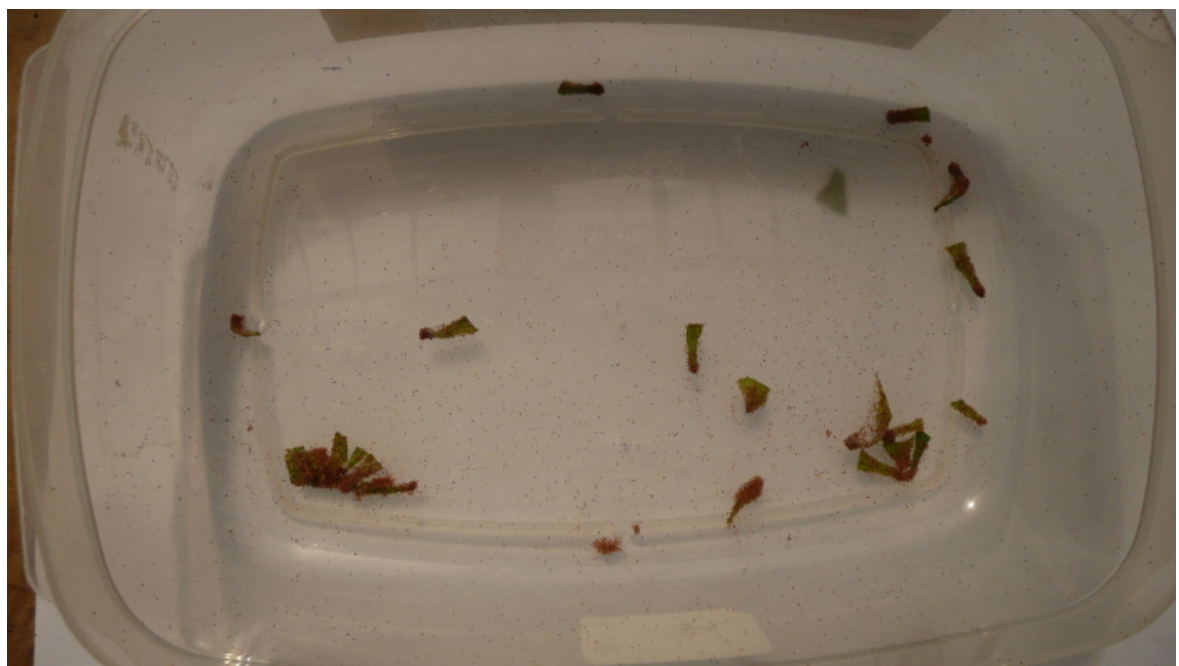

Figure 6. TSSM masses on bean leaf tips placed inside the pan. When sufficient number of trifoliate bean leaf tips with TSSM were found in the test plants, they were removed and placed inside the pan. These samples were used to infest treatment categories: light, medium and heavy which received 3, 20 and 40 masses of TSSM, respectively. Please click here to view a larger version of this figure.

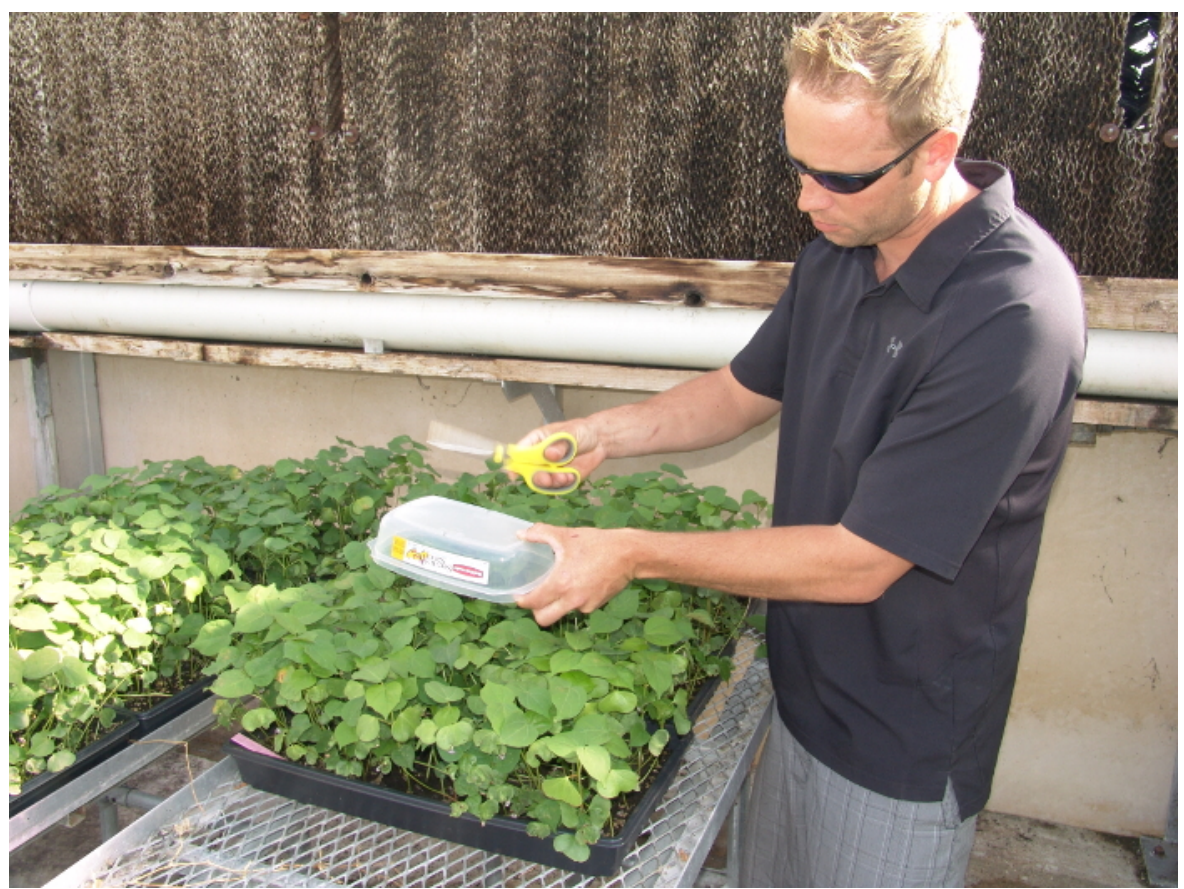

Figure 7. Turning pan upside down. The pans containing trifoliate bean leaf tips with TSSM were turned upside down on cotton canopy to infest the test plants. Please click here to view a larger version of this figure.

\section{Scan TSSM Infested Cotton Plants with the Multispectral Optical Sensor}

1. Horizontally mount the optical sensor onto the greenhouse frame approximately $7 \mathrm{ft}$ above the floor as shown in Figure 8. Set the distance between the scanner and the plant canopy at 36". Use a carpenter's level to ensure that the sensor is horizontally level.

2. Place uninfested trays of cotton plants on a wheeled push cart.

3. Activate the sensor switch and slowly push the cart under the sensor until the tray completely passes the sensor head as shown in Figure 8. Turn off switch. Retract the cart.

4. Repeat Step 3.3 three times, for a total of 3 replications

5. Repeat this procedure for all trays of cotton.

6. Repeat scanning on Day 1, Day 5, Day 6, Day 7, Day 9, Day 10, Day 12, Day 13 and Day 14 after treatment (DAT). The scanning provided the NDVI (Normalized Difference Vegetation Index) values ${ }^{12}$. Transmit the NDVI values to and store on a pocket PC which then can be downloaded to a computer in text format.

NOTE: NDVI was calculated from the following equation: NDVI = (NIR - RED)/(NIR + RED), where RED and NIR are the spectral reflectance values (0-255) in the red and near-infrared spectrums at 660 and $770 \mathrm{~nm}$, respectively. 


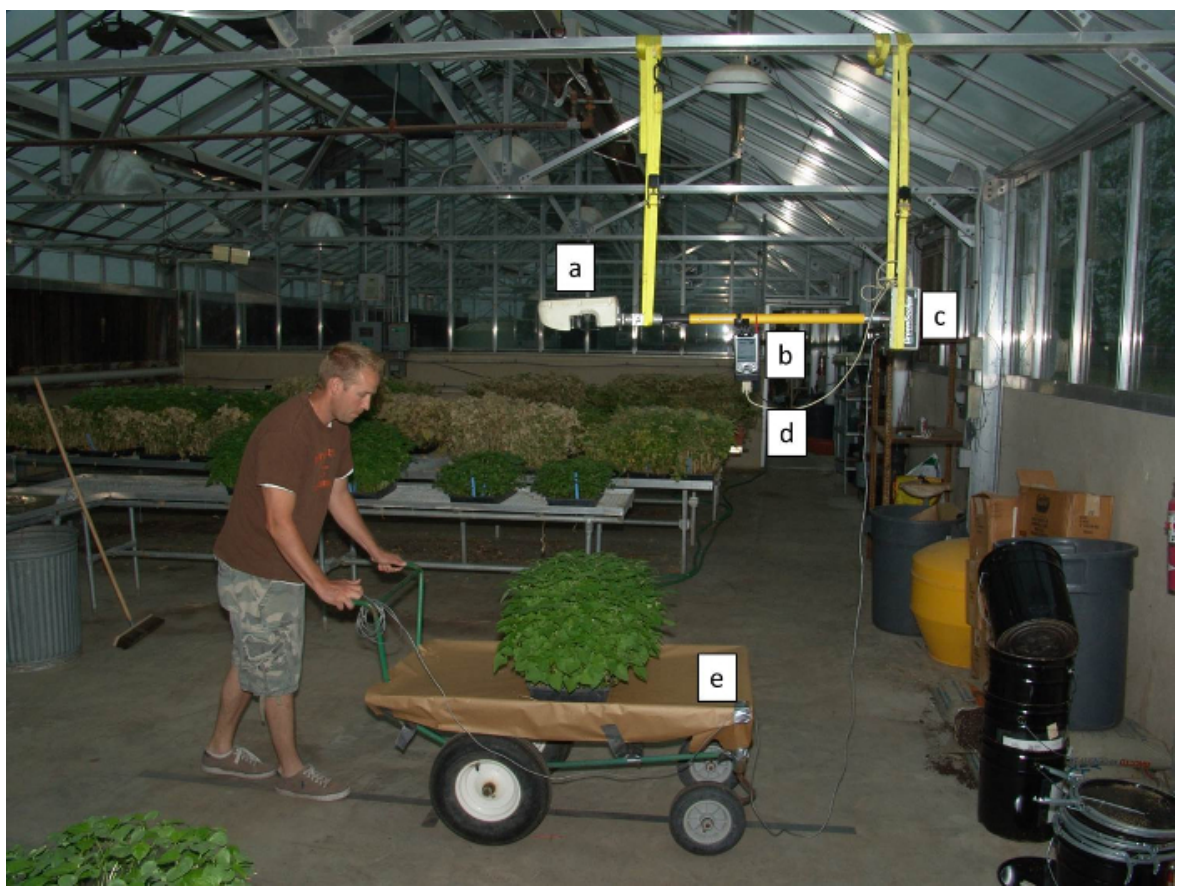

Figure 8. A multispectral optical sensor used to quantitatively measure the health of cotton plants infested with varying density levels of TSSM. The wheeled push cart with the test plants were slowly traversed under the sensor head to obtain spectral reflectance values. $\mathbf{a}$ is the sensor head; $\mathbf{b}$ is pocket PC; $\mathbf{c}$ is battery compartment and input/output ports; $\mathbf{d}$ is RS-232 serial data cable and $\mathbf{e}$ is the craft paper to provide uniform background. Please click here to view a larger version of this figure.

\section{Data Analyses}

1. Obtain the maximum NDVI values using the Proc Means procedure ${ }^{13}$. Calculate the percent reduction in NDVI values for each day of observation using Day 0 as a reference value. Analyze the data using the repeated measures PROC GLM procedure ${ }^{13}$.

NOTE: Means were separated using the Duncan's Multiple Range Test at $P=0.05$. Means with the same lower case letters were not significantly different.

2. Perform graphical illustrations of the data ${ }^{14}$ as shown in Figure $\mathbf{9}$.

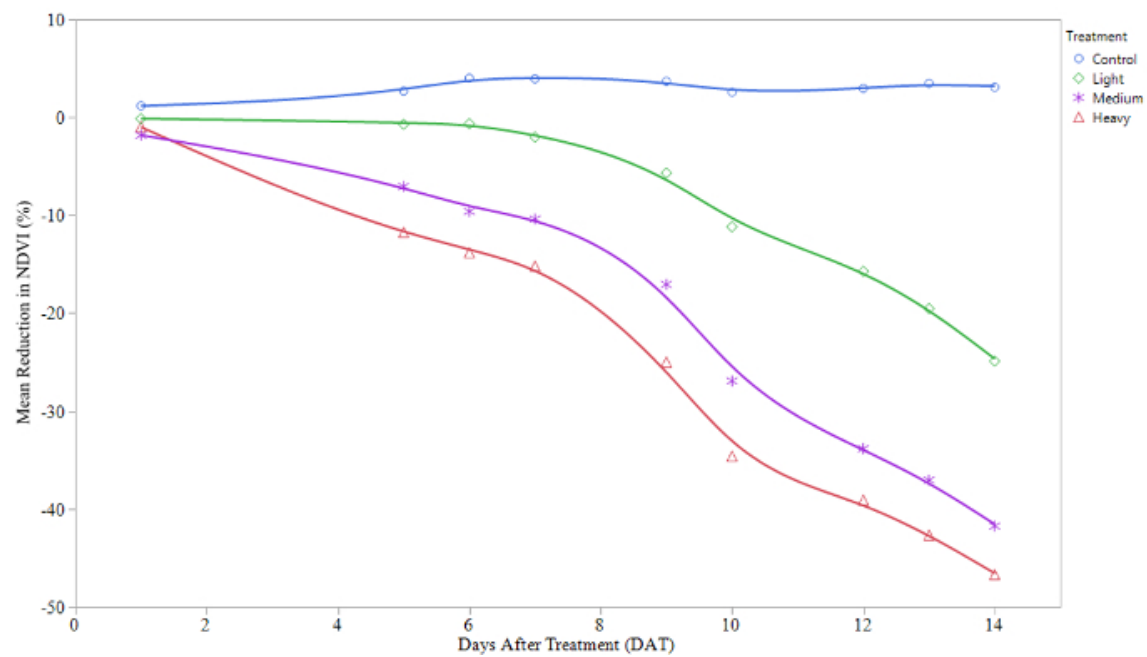

Figure 9. Percent reduction or change in NDVI relative to days after treatment. JMP software was used to graphically illustrate the functional relationship between percent change in NDVI relative to days of sampling (DAT). Please click here to view a larger version of this figure. 


\section{Representative Results}

The sensor emits red and infrared light and this light in turn gets reflected back from the plant canopy. The reflected light serves as a quantitative measurement of the visible and the near-infrared bands of the electromagnetic spectrum and is numerically recorded as NDVI (Normalized Difference Vegetation Index) reading. The NDVI values vary from 0 to 0.99 . The higher the NDVI reading the healthier the plant canopy. Healthy vegetation absorbs visible light and reflects the near-infrared light and unhealthy vegetation reflects more visible light and less near-infrared light. NDVI serves as a surrogate to photosynthetic activity and this spectral property is highly correlated to photosynthetically active radiation ${ }^{15,16,17,18}$ Very low values of NDVI ( 0.1 and below) corresponds to barren areas of rock or sand, while moderate values $(0.2$ to 0.3$)$ represent shrub and grassland and high values ( 0.6 to 0.8$)$ indicate temperate and tropical vegetation. The reflectance measurements were obtained under daytime lighting in the red and near-infrared regions of the spectra.

NDVI was calculated from the following equation: NDVI = (NIR - RED)/(NIR + RED), where RED and NIR are the spectral reflectance values $(0-255)$ in the red and near-infrared spectrums at 660 and $770 \mathrm{~nm}$, respectively. The sensor records reflectance values every $100 \mathrm{msec}$. The maximum NDVI readings culled from an array of NDVI values taken each time by the sensor were used in the analyses to minimize background reflectance and to provide consistently repeatable numerical values.

The analysis of variance of the data revealed that significant differences in percent reduction in NDVI were observed between lightly, medially and heavily infested cotton plants compared to the untreated control $(F=436.4 ; P<0.0001 ; d f=3,32)$. The NDVI values which described plant vigor during the test period varied significantly between days of observation $(F=1398.2 ; P<0.0001 ; d f=8,256)$. Also, the percent reduction in NDVI values were inversely correlated to the treatments during the observation period (DAT), but seldom tended to deviate from this pattern and interacting significantly with DAT $(F=201.5 ; P<0.0001$; $d f=24,256)$. The MANOVA test criteria for no DAT effect was significant as well (Wilk's $\lambda=0.00913 ; \mathrm{F}=339.0 ; \mathrm{P}<0.0001 ; \mathrm{df}=8,25)$. Similarly, the interaction between DAT and treatment was significant $($ Wilk's $\lambda=0.00101 ; \mathrm{F}=29.8$; $\mathrm{P}<0.0001 ; \mathrm{df}=24,73)$.

Figure 9 shows percent change in plant vigor as demonstrated by NDVI values during the observation period. A positive percent change in NDVI value indicates healthy growing plants, while a negative value indicates that the vigor of the plant has decreased since the first measurement (i.e. Day 0) was made. The non-infested control plants showed increased vegetative growth throughout the course of the study, while the TSSMinfested plants showed degradation in health over time. Mean separation of the treatments shown in Table 1 reveals that no definable difference in percent reduction in NDVI between treatment categories (light, medium and heavy) and the control was observed until Day 5 when infestation classes significantly deviated from the control and remained overwhelmingly so thereafter. This data demonstrates that the optical sensor can be effectively used in lieu of labor-intensive manual sampling to assess treatment efficacy against acaricides on cotton.

\begin{tabular}{|c|c|c|c|c|c|c|c|c|c|}
\hline \multicolumn{10}{|c|}{ Days After Treatment (DAT) } \\
\hline $\begin{array}{l}\text { Infestation } \\
\text { Category }\end{array}$ & 1 & 5 & 6 & 7 & 9 & 10 & 12 & 13 & 14 \\
\hline Control & $1.18 \pm 0.33 a$ & $2.70 \pm 0.40 a$ & $4.0 \pm 0.36 a$ & $3.94 \pm 0.37 a$ & $3.68 \pm 0.53 a$ & $2.57 \pm 0.42 a$ & $2.96 \pm 0.47 a$ & $3.48 \pm 0.38 a$ & $3.08 \pm 0.22 a$ \\
\hline Light & $-0.13 \pm 0.13 b$ & $-0.71 \pm 0.29 b$ & $-0.65 \pm 0.28 b$ & $-2.02 \pm 0.47 b$ & $-5.68 \pm 0.72 b$ & $\begin{array}{l}-11.17 \\
\pm 0.94 b\end{array}$ & $\begin{array}{l}-15.73 \\
\pm 1.76 b\end{array}$ & $\begin{array}{l}-19.54 \\
\pm 1.68 b\end{array}$ & $-24.9 \pm 1.90 b$ \\
\hline Medium & $-1.83 \pm 0.42 c$ & $-7.06 \pm 0.63 c$ & $-9.61 \pm 0.53 c$ & $\begin{array}{l}-10.39 \\
\pm 0.57 c\end{array}$ & $\begin{array}{l}-17.06 \\
\pm 0.80 \mathrm{c}\end{array}$ & $\begin{array}{l}-26.92 \\
\pm 0.72 c\end{array}$ & $\begin{array}{l}-33.84 \\
\pm 0.96 c\end{array}$ & $\begin{array}{l}-37.05 \\
\pm 1.14 \mathrm{c}\end{array}$ & $\begin{array}{l}-41.74 \\
\pm 0.73 \mathrm{c}\end{array}$ \\
\hline Heavy & $\begin{array}{l}-0.97 \\
\pm 0.58 \mathrm{bc}\end{array}$ & $\begin{array}{l}-11.76 \\
\pm 0.29 d\end{array}$ & $\begin{array}{l}-13.83 \\
\pm 0.86 \mathrm{~d}\end{array}$ & $\begin{array}{l}-15.20 \\
\pm 0.63 d\end{array}$ & $-25.0 \pm 1.0 d$ & $\begin{array}{l}-34.63 \\
\pm 0.54 d\end{array}$ & $\begin{array}{l}-39.07 \\
\pm 0.94 \mathrm{~d}\end{array}$ & $\begin{array}{l}-42.68 \\
\pm 0.62 \mathrm{~d}\end{array}$ & $-46.71 \pm 0.63$ \\
\hline
\end{tabular}

Table 1: Percent reduction in Max NDVI after cotton plants were infested with varying number of clusters or masses of TSSM. Cotton plants grown in plastic trays in the greenhouse were infested with three categories of spider mite density. Category Light received 3 masses or clusters of TSSM per tray, Category Medium received 20 masses per tray and Category Heavy received 40 masses per tray. Means were separated from control according to Duncan's Multiple Range Test $(P=0.05)$. Means followed by the same lower case letter were not significantly different at the $5 \%$ level of probability.

\section{Discussion}

Conventionally, insecticide efficacy tests conducted in the field include several treatments of the chemical applied at different rates and compared to an untreated check. Acaricides with varying toxicity profiles against instar and adult stages of TSSM are assessed to determine whether the damage caused by them could be reduced by the chemical treatment. TSSM samples are collected and brought to the laboratory where they are examined under the microscope and the varying stages of TSSM are counted and recorded. It is critically important to take adequate samples of the host plant to determine the damage in each treatment and differentiate them with statistically acceptable precision. The number of samples that are required to differentiate the treatments from each other depends upon the distribution profile of the organism. Highly patchy distribution of the TSSM leads to a considerable amount of variation between sample areas, and many plants must be sampled in order to ensure reproducibility of the population estimates. However, budget, manpower, time and statistical precision are important factors that impinge upon sampling techniques. It behooves the researcher to allocate the available resources optimally to conduct sampling with the least cost but with most precision.

Entomologists in lieu of counting TSSM stages visually score the damage based upon a scale of no damage to varying levels of damage. For instance, some researchers proposed binomial sampling, where only the proportion of infested leaves was scored rather than the number of spider mites per leaf ${ }^{9,19}$. Others estimated damage by TSSM on cotton based on a leaf reddening index scale, which varied from stippling 
and reddening to extensive reddening of vegetation canopy ${ }^{19}$. These methods are arbitrary, anecdotal and biased by individual perceptions of the degree of damage. A more robust and quantitative assessment of the damage caused by TSSM is required to evaluate and separate the treatments with statistical precision.

The ground-based multispectral optical sensor appears to be an improved sampling tool to quantitatively determine the damage inflicted by TSSM and to separate the treatments more precisely than the visual damage scoring system adopted by many researchers. However, researchers have reported that the data intensive hyperspectral remote sensing provided numerous spectral signatures to identify and detect crop stresses and canopy characteristics compared to multispectral remote sensing which is less data intensive with two wave lengths ${ }^{20,21}$. Using a hyperspectral spectrometer, Reisig and Godfrey found that the NIR reflectance wavelength $\approx 850 \mathrm{~nm}$ as an informative spectrum in distinguishing arthropod-infested from uninfested cotton ${ }^{22}$. In this study, we have shown that the multispectral reflectance values (NIR value being $\approx 770 \mathrm{~nm}$ ) with just two spectral bands were able to identify and characterize cotton plants infested with varying densities of TSSM. Also, we have reported earlier that the multispectral optical sensor not only effectively separated cotton plants infested with widely varying density categories of TSSM, but also showed that spiromesifen was more effective than abamectin in controlling TSSM in early season cotton in the greenhouse at one-half rate of the lowest label rate $\mathrm{e}^{23}$.

The multispectral optical sensor could be mounted on a mobile research platform and the reflectance values could be obtained from the treated areas of the vegetation canopies without human subjectivity. Acaricidal efficacy data can thus be obtained without much human labor. The NDVI data can be easily uploaded to the computer and analyzed using commercial statistical software. A GPS receiver also can be mounted onto the mobile platform to collect the GPS coordinates in order to generate a damage map of the field. Using multispectral reflectance signatures from the plant canopy, the multispectral optical sensor provides a rapid and cost effective means of identifying and quantifying plant stress. Moreover, a much larger area of the field can be covered in less time with higher spatial resolution of plant canopy compared to conventional field scouting. It is important to remember that the threshold damage level for TSSM on cotton is variable from region to region in the United States. For example, TSSM damage would be higher in an arid environment such as in California compared to the Midsouth region where rainfall and high humidity often prevail ${ }^{24}$. Therefore, yield loss due to TSSM damage will be variable and so is the damage threshold. However, reports from Mississippi, Arkansas and Tennessee reveal that the threshold level for TSSM on cotton appears to be when 30 to $50 \%$ of the plants are infested and populations are actively increasing. Furthermore, field grown plants are exposed to multiple stresses including water stresses and feeding of arthropod herbivores and the interactions between these activities can substantially reduce plant productivity and are likely to influence damage threshold. The TSSM can reduce stomatal conductance, photosynthesis and transpiration rate in cotton ${ }^{25}$. Plants grown in the greenhouse are influenced by UV light radiation and it does significantly influence stomatal function, photosynthesis and canopy morphology ${ }^{26,27}$ and could probably have an additive effect on plant stress. However, TSSM is capable of avoiding UV radiation by having access to habitat protected from solar radiation on the lower surface of the plant canopy ${ }^{28,29,30}$, where it resides.

The height of the optical sensor above the target canopy and the orientation of the sensor relative to the target are important factors that affected significantly the reflectance values obtained by the multispectral optical sensor ${ }^{31}$. For instance, when the mobile research platform traverses through a row crop field such as when cotton canopy is open, the sensor is likely to produce different results depending upon the orientation of the sensor, either parallel to or perpendicular to the row. It is also likely that soil and other background materials may greatly influence the sensor readings, especially when the sensor is oriented perpendicular to the row. In order to obtain maximum response from the sensor, the sensor head should be oriented in-line with and directly above the rows. Although orienting the light beam perpendicular to the rows is more likely to pick up background soil reflectance, this may be acceptable, however, when cotton canopy is closed with lush vegetation. In addition, operators should follow manufacturer's recommendations on an operating height range of $81-122 \mathrm{~cm}$ and orienting the sensor head in-line with the target to obtain maximum signal response. It is important to charge the sensor battery before use or it should be kept plugged in to avoid troubleshooting. Low battery level is likely to produce erroneous readings.

\section{Disclosures}

The authors have nothing to disclose.

\section{Acknowledgements}

We appreciate the assistance of Chris Parker who daily scanned the plants and Curtis Hubbard who maintained the plants in the greenhouse

\section{References}

1. Hoy, M. A. Agricultural acarology: Introduction to integrated mite management. Vol. 7 CRC Press (2011).

2. Jeppson, L. R., Keifer, H. H., \& Baker, E. W. Mites injurious to economic plants. Univ of California Press (1975).

3. Brandenburg, R., \& Kennedy, G. Ecological and agricultural considerations in the management of twospotted spider mite (Tetranychus urticae Koch). Agric. Zool. Rev. 2 185-236 (1987).

4. Saito, Y. The concept of "life types" in Tetranychinae. An attempt to classify the spinning behaviour of Tetranychinae. Acarologia. 24 (4), 377-391 (1983).

5. Gore, J. et al. Impact of two-spotted spider mite (Acari: Tetranychidae) infestation timing on cotton yields. Journal of Cotton Science. $1734-39$ (2013).

6. Adamczyk, J. J., Jr., \& Lorenz, G. M. in Beltwide Cotton Conference. 981-1000 National Cotton Council, Memphis, TN (2016).

7. Williams, M. R. in Beltwide Cotton Conference. 1013-1057 National Cotton Council, Memphis, TN (2016).

8. Van Leeuwen, T., Vontas, J., Tsagkarakou, A., Dermauw, W., \& Tirry, L. Acaricide resistance mechanisms in the two-spotted spider mite, Tetranychus urticae and other important Acari: A review. Insect Biochem Mol Biol. 40 (8), 563-572 (2010).

9. Wilson, L., \& Morton, R. Seasonal abundance and distribution of Tetranychus urticae (Acari: Tetranychidae), the two spotted spider mite, on cotton in Australia and implications for management. Bull Entomol Res. 83 (02), 291-303 (1993). 
10. Fernandez, F., Gepts, P., \& Lopez, M. Stage of development of the common bean plant. Communication Information Support Unit edn, 32 CIAT (1986).

11. Clotuche, G. et al. The formation of collective silk balls in the spider mite Tetranychus urticae Koch. PLoS. ONE. 6 (4), 1804-1807 (2011).

12. Rouse Jr, J. W., Haas, R., Schell, J., \& Deering, D. Monitoring vegetation systems in the Great Plains with ERTS. NASA special publication. 1 (SP-351), 309-317 (1974).

13. Institute Inc., Cary, NC., (2012).

14. Institute Inc., Cary, NC., (2013).

15. Asrar, G., Fuchs, M., Kanemasu, E., \& Hatfield, J. Estimating absorbed photosynthetic radiation and leaf area index from spectral reflectance in wheat. Agron J. 76 (2), 300-306 (1984).

16. Myneni, R. B., \& Hall, F. G. The interpretation of spectral vegetation indexes. Geoscience and Remote Sensing, IEEE Transactions on. 33 (2), 481-486 (1995).

17. Sellers, P. J. Canopy reflectance, photosynthesis and transpiration. Int J Remote Sens. 6 (8), 1335-1372 (1985).

18. Tucker, C. J. et al. Higher northern latitude normalized difference vegetation index and growing season trends from 1982 to 1999 . Int. J. Biometeorol. 45 (4), 184-190 (2001).

19. Wilson, L. et al. Within-plant distribution of spider mites (Acari: Tetranychidae) on cotton: a developing implementable monitoring program. Environ Entomol. 12 (1), 128-134 (1983).

20. Fitzgerald, G. J., Maas, S. J., \& Detar, W. R. Spider mite detection and canopy component mapping in cotton using hyperspectral imagery and spectral mixture analysis. Precision Agriculture. 5 (3), 275-289 (2004).

21. Herrmann, I. et al. Spectral monitoring of two-spotted spider mite damage to pepper leaves. Remote Sensing Letters. 3 (4), $277-283$ (2012).

22. Reisig, D., \& Godfrey, L. Spectral response of cotton aphid-(Homoptera: Aphididae) and spider mite-(Acari: Tetranychidae) infested cotton: Controlled studies. Environ Entomol. 36 (6), 1466-1474 (2007).

23. Martin, D. E., Latheef, M. A., \& López, J. D. Evaluation of selected acaricides against twospotted spider mite (Acari: Tetranychidae) on greenhouse cotton using multispectral data. Exp Appl Acarol. 66 (2), 227-245 (2015).

24. Boudreaux, H. B. The effect of relative humidity on egg-laying, hatching, and survival in various spider mites. J Insect Physiol. 2 (1), 65-72 (1958).

25. Bondada, B., Oosterhuis, D., Tugwell, N., \& Kim, K. Physiological and cytological studies of two spotted spider mite, Tetranychus urticae K., injury in cotton. Southwest Entomol. 20 (2), 171-180 (1995).

26. Teramura, A. H. Effects of ultraviolet B radiation on the growth and yield of crop plants. Physiol Plant. 58 (3), $415-427$ (1983).

27. Teramura, A. H., \& Sullivan, J. H. Effects of UV-B radiation on photosynthesis and growth of terrestrial plants. Photosynthesis Res. 39 (3), 463-473 (1994).

28. Ohtsuka, K. Deleterious effects of UV-B radiation on herbivorous spider mites: they can avoid it by remaining on lower leaf surfaces. Environ Entomol. 38 (3), 920-929 (2009).

29. Sakai, Y., \& Osakabe, M. Spectrum-specific damage and solar ultraviolet radiation avoidance in the two-spotted spider mite. Photochem Photobiol. 86 (4), 925-932 (2010).

30. Suzuki, T., Watanabe, M., \& Takeda, M. UV tolerance in the two-spotted spider mite, Tetranychus urticae. J Insect Physiol. 55 (7), $649-654$ (2009).

31. Martin, D. E., López Jr, J. D., \& Lan, Y. Laboratory evaluation of the GreenSeeker handheld optical sensor to variations in orientation and height above canopy. International Journal of Agricultural and Biological Engineering. 5 (1), $43-47$ (2012). 